\title{
Option Pricing with the Credit Risk in Incomplete Markets
}

\author{
Zhaohai WANG \\ Department of mathematics and statistics, Ankang University, Ankang, Shanxi 725000, China \\ akwzh@163.com
}

\begin{abstract}
The paper considers the financial derivatives model with the credit risk. If the market is incomplete, we though the value of corporation value model, the credit risk will be introduced to options pricing. First of all, the general valuation formula in given about the Europe option under the condition of incomplete market and default risk .Second, the use of Black----Scholes the risk-neutral option pricing for reference, application of martingale pricing and probability methods, The research work of paper will be helpful to enrich the study derivatives pricing with credit risk.
\end{abstract}

Index Terms - Credit risk, Incomplete market, Hedging

\section{Introduction}

If the market is incomplete, we have several choices of equivalent martingale measures to price contingent claims. Credit risk is increasingly being recognised as a significant area of risk and arbitrage, yet there exists relatively little research on it. In this paper we show that operational risk represents a fundamental risk to option hedging and investigate it by proposing a new theoretical model. We derive an exposure indicator for the operational risk of option hedging[1] and the resulting operational risk distribution. We obtain analytical results for various risk measures including the Value at Risk equation; this includes deriving a new analytical result for the option function of the half-normal distributions (which will be of interest to Statisticians in general). We determine an analytical solution to the price of options under operational risk. We conduct numerical experiments on empirical option data to validate our model and estimate the operational Value[2] at Risk for option hedging.

Recall that we didn't impose conditions on the preferences of the economic agents other than that they prefer more to less. There exists currently a good deal of literature on hedging methods for the pricing options. It is well known that in this case a straightforward Monte Carlo simulation algorithm will be time-consuming and yield unstable results for the prices and especially the sensitivities. The knock-in/out features in the barrier option payoffs lead to slower convergence of the Monte Carlo algorithm. To address this problem the following (semi-)analytical approaches have been developed for specific models.

In any case, theoretically, long-run uncertainty about demographic changes and shocks restricts the land supply and raises the land price. Now, the spatial demographic risk is local by nature: a shortfall in one place entails a surplus somewhere else; therefore, it is an insurable risk.
Consequently, a future insurance market may be a tool with which to improve the way the housing market works. Empirically, we conduct the analysis for a French department. The data base used in this study is made up of individual transactions for developable land for residential purposes $(19,495$ observations) or secondary or tertiary activities (1,667 observations) between 1989 and 2002.The results show that, during the upward trend in the land market, the price of developable land is significantly higher when the change in population in the vicinity is more volatile, and so more difficult to predict. The plot price is $7.8 \%$ (housing market) or $15.3 \%$ (office and factory market) higher when population change in the 10 nearest communes increases by one standard deviation.

\section{Option Pricing Theory}

We compare our results with those given by Merton [3].We first explain Merton's results. Merton restricted his discussion to discrete probability distributions. Let an asset have terminal value $x_{i}$ with probability $f\left(x_{i}\right)$, where the values of $x_{i}$ with positive probability are $x_{1}<x_{2}<\cdots x_{m}$.In addition to the two well-known definitions of increase in risk, namely, a mean-preserving spread and Rothschild and Stiglitz increase in risk, he pro-posed two more definitions of changes in risk which are called pointwise riskier and extremum riskier, respectively. The following are the two definitions.

Definition 2 (Merton.[3]): A distribution $\mathrm{g}(\mathrm{x})$ is said to be pointwise riskier than distribution $\mathrm{f}(\mathrm{x})$ if $\mathrm{s}(\mathrm{x}) \equiv \mathrm{g}(\mathrm{x})-\mathrm{f}(\mathrm{x})$ is a mean-preserving spread whosesupport contains the support of the original distribution $\mathrm{f}(\mathrm{x})$.

Definition3(Merton[3): Adistribution $\mathrm{g}(\mathrm{x})$ is said to be extremum riskier than distribution $f(x)$ if the cumulative distribution $\mathrm{G}(\mathrm{x})$ is strictly larger thanF(x) at the immediate right of the left border of the support of $f(x)$ and is strictly smaller than $F(x)$ at the immediate left of the right border of the support of $\mathrm{f}(\mathrm{x})$. Rasmusen's Proposition 1 states that a mean-preserving increase in risk does not reduce any option values, which is Merton's (1973) Theorem .His Proposition 1a states that a mean-preserving increase in risk strictly increases[4]all option values, which he proved to be false. His Proposition $1 \mathrm{~b}$ states that a mean-preserving increase in risk strictly increases some option values and reduces no option

Foundation items: Natural Scicence Foundation of Ankang Univercity(No, 2013AYPYZR03)

About the author: Zhaohai wang (1966 -), Associate Professor, Research direction: Option Pricing; Optimization; Fuzzy reasoning; Risk Analysis. 
values. These two results are relatively straightforward. Thus, we focus on his main results.

\section{Option Pricing in Incomplete Markets}

\subsection{Option pricing with Hedging}

This general mean variance hedging approach does not take into account additional information on market prices. In this paper we assume the existence of such additional market information, which is represented by a prescribed, finite set of observed prices of different contingent claims. These specific contingent claims have to be non-attainable or non-replicable by dynamic portfolio strategies in order to deliver new, relevant information on the underlying price system of the market. Due to no-arbitrage arguments, the set of all possible linear price systems or equivalent martingale measures shrinks and we have to consider a modified mean-variance hedging problem, which allows for buying or selling specific contingent claims at the observed prices.

We would like to present an alternative approach of deriving the Black-Scholes equation for the option pricing model, by which the argument of risk neutrality can be explained in a more succinct manner (Cox and Ross[7],1976). Suppose we write the stochastic process followed by the option price as

$$
\frac{d c}{c}=\mu d t+\sigma d Z
$$

Where $\mu$ is the expected rate of return of $\mathrm{c}$ and $\sigma^{2}$ the corresponding variance of the rate of return.

$$
d c=\left(\frac{\partial c}{\partial t}+\mu S \frac{\partial c}{\partial S}+\frac{1}{2} \frac{\partial^{2} c}{\partial S^{2}} \sigma^{2} S^{2}\right) d t+\sigma S \frac{\partial c}{\partial S} d Z
$$

The above statement of risk neutrality can be represented mathematically as $C=C(S, t)=e^{-r \tau} E\left[\max \left(S_{T}-K, 0\right)\right]$.The option pricing model takes the following form

$$
C=S N\left(d_{1}\right)-K e^{-r \tau} N\left(d_{2}\right) \text { Where }
$$

$$
\begin{aligned}
& d_{1}=\frac{\ln (S / K)+\left(r+\frac{\sigma^{2}}{2}\right) T}{\sigma \sqrt{T}}, d_{2}=d_{1}-\sigma \sqrt{\tau}=\frac{\ln (S / K)+\left(r-\frac{\sigma^{2}}{2}\right) \tau}{\sigma \sqrt{\tau}}, \\
& \tau=T-t, \sigma=\sqrt{\operatorname{var}(d S / S)} .
\end{aligned}
$$

The above call price formula can be interpreted using the language of probability. First, $N\left(d_{2}\right)$ is been as the probability of the call option being in-the-money at expiry and so $K N\left(d_{2}\right)$ can be interpreted as the risk neutral expectation of the payment made by the holder of the call option at expiry on exercising the option. Hence, the expectation of the call value at expiry is $S e^{r \tau} N\left(d_{1}\right)-K N\left(d_{2}\right)$, which is then discounted by the factor $e^{-r \tau}$ in the risk neutral world to give the present value of the call price.

\subsection{The Option Pricing in Incomplete Markets}

To model short-term option values, we look at 'pure' price variability over time, that is, by controlling a set of variables $X$ that affect this price and vary over the course of time. The starting equation is $d p=\alpha_{1} d t+\sigma \varepsilon_{t} \sqrt{d t}$, where $\mathrm{P}$ is the change in the price of developable land over the period $\mathrm{dt}$ under the 'pure' influence of time, that is, having expurgated factors of variation included in $\mathrm{X}$ and where $\varepsilon$, is a random variable of zero mean and of unit variance; $\alpha$ is the

trend and $\sigma^{2}$ the price variance. $\mathrm{P}$ is estimated by a randomeffects model

$$
P_{i j t}=X_{i j t} b+b_{t} T+v_{t}+\varepsilon_{j}+\varepsilon_{i j t}
$$

Where the price $P_{i j t}$ of transaction $\mathrm{i}$ in commune $\mathrm{j}$ and in the period $\mathrm{t}$ is explained by a set of variables $\mathrm{X}$, by a continuous time variable $\mathrm{T}$ capturing the trend, by a random variable $v_{t}$ that is dependent on the period preceding $\mathrm{t}$, a second random variable depending on the commune $\mathrm{j}$ and by an individual error $\varepsilon_{i j t}$. The variance of $v_{t}$ is then introduced into the explanatory model of land price where $\sigma_{n}$ is the standard deviation of variation of population change in the communes around $\mathrm{j}$ within a neighborhood $\mathrm{w}$ and $\Delta_{n}$ is the change in population over this period. This standard deviation is calculated directly from population censuses. The standard deviation of population on which households' base their decision to purchase has to be observed over a long enough period.

\section{Option Pricing Theory under Additional Market}

We consider the financial market of the previous section, but under the assumption of additional market information, which is represented by a given, finite set of at time 0observed $\mathrm{T}$-contingent claim prices.

The price of Incomplete Markets follows a geometric Brownian motion in the Black-Scholes model. If the finance market is complete this paper gives an accurate hedging strategy by another method. Then we introduce a dynamic measure of risk to the incomplete market, under which we have acquired the optimal replication of a contingent claim in the finance market which is induced by a risk neutral probability. With an application of a generalized Clark formula[5]the paper provides the optimal hedging strategy for a contingent claim. The preferred spelling of the word "acknowledgment" in America is without an " $\mathrm{e}$ " after the " $\mathrm{g}$ ".

To illustrate the flexibility of the method we implemented the algorithm for a number of models, including local volatility models with jumps and models with time-dependent jumpdistributions. In the cases of the diffusion and jump-diffusion models where results had been obtained before in the literature, the algorithm produced outcomes that accurately matched those results, and we numerically investigated the order of decay of the error. 
We derived a theoretical upper bound for the error of the outcomes produced by the algorithm that is linear in the spatial mesh size and the truncation error. We showed that

an additional logarithmic factor may arise in this error bound when the Hull[6] density has a pole of order two at the origin. In addition, this bound is also linear in the time mesh size if the model is time-inhomogeneous. Numerical experiments suggest that for a number of models the error of the outcomes generated by the algorithm actually decays in the spatial mesh size. It would be of interest to establish error bounds under weaker regularity assumptions, and obtain sharp rates of convergence for the specific models, which is a topic left for future research. Although in principle the method also applies to higher-dimensional Markov processes, the size of the generator matrix would make straightforward application of the algorithm computationally infeasible. The investigation of efficient extensions of the approach to Markov processes of moderate dimension is another topic left for future research.

\section{Conclusions}

Several kinds of pricing formula of European foreign currency option are obtained. Under different assumptions, the option include two styles: the first is the pricing model where the domestic and foreign bond rates are underlying asset and assumed stochastic, using the two approach of martingale theory and partial different equation, the pricing formula of this option is established which exchange rates, bond rates follow lognormal diffusion process. The second is the pricing model where the domestic and foreign interest rates are underlying asset and assumed stochastic, using the method of forward variable translation which is motivated by forward measure is martingale theory. As a consequence, the set of equivalent martingale measures to be considered in the selection problem of the previous section shrinks to the set of admissible equivalent martingale measures.

\section{References}

[1] F.Black, M.Scholes. The pricing of options and corporate liabilities. Journal of Political Economy, vol. 81, pp.637-659, April 1973.

[2] F.Black and P..Karasinski. Bond and option pricing when short rates are lognormal. Financial Analysts Journal, vol.7, pp.52-59, 1991.

[3] Merton R. Option pricing when underlying stock returns are discontinuous. Journal of Financial Economics, vol. 3 pp.125-144, 1976.

[4] F.Black, E.Toy. A one-factor model of interest rates and application to treasury bond options. Financial Analysts Journal, vol.6, pp.33-39, 1990..

[5] S.M.Schaefer, E.S.Schwartz. A two-factor model of the term structure: An approximate analytical solution. Journal of Financial and Quantitative Analysis, vol.9 (19), pp.413-424, 1984.

[6] J.Hull and A.White. Bond option pricing based on a model for the evolution of bond prices. Advances in futures and options Research, vol.6: pp.1-13,1993. 